\title{
The Fluid Traditional and Indigenous Structure of Climatism in Physical Architecture and Its Role in Hospital Construction
}

\author{
Sania Sami' ${ }^{1}$, Roughayeh Rezanejad Zanjany² \\ ${ }^{1}$ Department of Architecture and Urban Planning, Science and Research Branch-Qeshm, Islamic Azad University, Qeshm, Iran \\ ${ }^{2}$ Department of Architecture and Urban Planning, Yadegar Imam, Shahr-e-Rey, Islamic Aza University, Tehran, Iran \\ Email: sania_sami@yahoo.co.uk
}

How to cite this paper: Sami, S. and Zanjany, R.R. (2020) The Fluid Traditional and Indigenous Structure of Climatism in Physical Architecture and Its Role in Hospital Construction. Journal of Building Construction and Planning Research, $\mathbf{8}$, 1-13.

https://doi.org/10.4236/jbcpr.2020.81001

Received: June 25, 2019

Accepted: January 6, 2020

Published: January 9, 2020

Copyright $\odot 2020$ by author(s) and Scientific Research Publishing Inc. This work is licensed under the Creative Commons Attribution International License (CC BY 4.0).

http://creativecommons.org/licenses/by/4.0/

\begin{abstract}
The issue of climatism is a matter of concern today, given the growth of technology and the subject of globalization, which is defined and explained in many respects. The rapid advancement of technology makes communication and navigation readily available. This factor causes the challenge for human societies to discover more recent developments that in turn raise the issues of how can the climatism be compatible with the creation of an architectural work, taking into account that the countries' conventional boundaries lose their importance. And, the factors affect climatism, in other words, the way that climatism, human societies with diverse cultures, and the surrounding environment interact with each other, is raised. And, in general, the way that an architecture work to interact with its environment is discussed. In this sense, the traditional and indigenous architecture, and the fluidity of the region in the architectural framework also address the characteristics of the physical and architectural features of each region from the architectural arena, introducing effective approaches to architecture and urban planning (objective and tactical approaches), using the rational-logical approach to regional review. Then, the discussion of regionalism and regional influences in the physical fabric of each traditional architectural structure's region is presented, with the special look of traditional architecture that is expressed in consistency between the building and the nature, and to explain the arguments to the examples and characteristics.
\end{abstract}

\section{Keywords}

Regionalism, Climatism, Native Architects, Fluidity Body, Structural Architects, Health Centers, Climatism in the Hospital 


\section{Introduction}

This issue has been examined by theoreticians such as Ozkan, Rudolf, Mumford, Newcomb, Harris, and Cole Cohen, with the differences and similarities expanded taking into account economic, social, regional, indigenous viewpoints to discuss the relationship between the climate and region with the traditional architecture, and analyze interaction between these factors on the human communities and peripheral environment and vice versa.

It is argued that, as there is an integrative order governing all universe with the whole being and all the components interact with each other in harmony, the issue of the effect of the architectural work and the environment climate still remains as a whole including its components, and in the further discussion, the issue of the hospital and the process of hospital construction are dealt with and the performance of these buildings is evaluated according to the climate, peripheral environment and social culture per region. The hospital and in general hospitals construction are not excluded, and human development in the medical field and the need for medical services caused the hospital buildings evaluations more often to help people step in these buildings with physical and spiritual security and on the other hand made the physical body of these buildings designed in such that meet all human needs taking into account various factors (economic, climate and location, efficiency of materials, efficiency, safety, etc.).

The current paper is essentially a mixed logical reasoning. Data are collected from available literature using objective and relative approaches to extract discussion and arguments applicable to the development of optimal solutions for designing a safe, climate-compatible hospital.

Traditional architecture, given its features, is an architecture that draws a certain cultural burden from a specific regional boundary handed over from generation to generation over time. All the buildings that have survived to the present day are classified as traditional structures; although this criterion is not enough precise and raises a lot of questions, the centrality of the issue of the relationship between the place architecture and the domination of thought, in a critical position relative to the forms and contexts resulting from the communities created by displacements and the global community, leads to the critical re-examination of native culture with modernist strategies. Also, the notion posed as a confirmation of native environments from the point of view of critical regionalism has a clear link with the global issue of ever-increasing environmental crises. In the context of the term regionalism, given the many common concepts, mainly in the field of definition and in the field of application, it is concluded that the main purpose of the term regionalism faces controversies and, in some cases, evident contradictions [1].

The regional attribute is the essential property of any architecture. Since all buildings are part of the herein defined, they cannot be the same everywhere, but they have to have certain specific locational features. From a long time ago, this feature was known as atmosphere, and historic buildings, although they have a 
lifestyle, usually have a definite local atmosphere. Thus, architecture helped man know himself with the spirit of a place and gave him a sense of belonging and safety [2]. Also, due to the fact that regionalism, in the heart of its definitions, has an interactive look at some bipolar words such as tradition-modernity, global-indigenous, and past-future, specific approaches will replace the regionalism approach in a situation where the balance between the two terms is eliminated [3].

\section{Physical Structures and Traditional Architecture Features}

In order to achieve the meanings and values of the traditional architecture, the reflection on the question "What is a tradition?" may help. The propagation of the tradition is holy, and what is traditional is inseparable from holiness. Tradition means the facts and principles with a divine origin reveled and inspired on human beings and the whole of the cosmic realm, and are the only truth that is the heart and the fullness of all truths. All traditions are earthly manifestations of patterns of heaven, ultimately attached to the same pattern of eternal tradition. Additionally, the tradition with this interpretation in Western civilization is widespread since the last stage of the deification of knowledge and the practice of modern mankind. Reconciliation of tradition is in this way created a universal parable, because the fact that the form of a lifelong human being was created in the course of the ages was almost lost and declined. (Seyyed Hossein Nasr)

\section{Regional Architect with Climatic Conditions (Traditional Architecture)}

In addition to a desirable appearance, the creation of a traditional structure that pays attention to valuable meaning, needs a specific architecture like traditional architecture. Traditional architect knows himself sacred, because he defined himself in a holy hierarchy through which it was connected to the architect of the world. The architects considered the Lord as the architect; building the dome of the universe, and created the whole world so beautiful and efficient with no inefficiency. Architecture may play the same role. He created the people of God building with a divine approach, and imitated the Divine Manor in order, efficiency, beauty and inaccessibility. The building can be so constructed that coordinated with universe architectural state and not only does not counteract the order of nature, but is considered part of it.

\subsection{Recognizing Gaia}

In a system habitat, with its physical resources, such as the climate, the shape of the earth, the soil, the biological resources (including plants, animals, and microorganisms, etc.) and a set of processes between them lead to habitat continuity. Thus, the human habitat system contains human and the environment affected by their presence. This habitat system includes the sustainability of a small system to a macro-scale system that is the earth. An approach with different scales to the environment and human activities in such thinking makes it possi- 
ble to maintain the sustainability of the smallest scales to the large scales, the entire earth. If the whole earth is defined as a single unit, then Earth can also be defined as a complex biological system that, by retrieving all its core systems, is a living organism, the Greek word Gaia is derived from the definition of James O'Lock of Earth, which considers Earth as a "whole integrated life" [4]; "an elemental approach to the whole, in which knowledge is developed in separate research fields", which makes such an attitude diminish the importance of what the whole involves.

\subsection{Sustainability of Regionalism and Nativism}

Referring to Rudolfsky's (1964) and Oliver (1969) and (1977) studies, Ozkan (1985) and (2007) to describe regionalism and nativism differences knows nativism with two Protective and Instructive perspectives and believes that although both groups ideal is to present a new and modern representation of forms and spatial organization of native architecture, they differ in the face of the two concepts of "technology" and "community". Also, Ozkan considers the major difference between regionalism and nativism in their scale of influence. In his opinion, when nativism or neo-nativism extends from the scale of small buildings to large urban buildings, attitudes toward them are limited. In other words, nativism is an aspect, and in fact, is a limited aspect of regionalism that has a limited form of construction, which is predominantly in native culture [5]. The semantic interplay between regionalism and nativism arises when industrialization and the emergence of new technologies, along with new ways of life, challenge human societies.

In contrast, Rudolf (1985), with this notion, uses regionalism instead of nativism. He expresses industrialization as a limit to regionalism. In his opinion, on the one hand, the ease of travel and communication limits regionalism, and on the other hand the increased cost of traditional materials and the skills associated with it are other limitations. Also, by stating that "some of the buildings in the region are more illustrative", introduces some types of buildings that have regional features such as the house, and also refers to some other ones hardly regional-oriented, such as hospitals, airports, or modern technical services (Mazhar Khan, 1985, 191). In this way, he limits the scope of regionalism to structures that include user-specific behavior patterns that surround the cultural aspects of their lives.

What explains the major difference between regionalism and nativism is the regionalism adoption from past architectural forms for responding to today's noble needs and their functional needs [6]. Mamford considered noble regionalism to recognize the real human needs in connection to the land, soil, climate, working conditions and hidden customs of neighborhoods; and believes that this is much more difficult than the adaptation of the past decoration. In his opinion, the close consistency of the building with the environment and the conscious effort for the optimal use of regional resources and processes are representations of regionalism [7]. He also believes that regionalism is not merely the use of all 
regional materials, or simply copying forms of construction that our ancestors did use, but regional forms are those that are intrinsically linked to real life and which are more successful in fostering people's feelings about their home and environment. They are not exclusively made from the soil, but represent the existing cultural conditions of the region [5].

Conversely, nativism, in terms of style renewation, views past architectural forms and prescribes a kind of emotional historicism in the face of past architectural traditions.

\section{Climate and Ethnicity in Construction Framework}

Regionalism is different from Ethnicism. Although both share the two geographic terms. Ethnicism, in the beginning, was functional in the $19^{\text {th }}$ and early $20^{\text {th }}$ centuries, and from a cultural point of view, negative perceptions were made about it, and pointed to things that were limited and natural. Ethnic works differed from region-specific works, as these works, due to the distance from the cultural center, were subject to limitations, especially in terms of standards. Whereas, on the contrary, regionalism, by virtue of its resistance to the identical values and tastes developed from the administrative centers, activated local values, and the regional look, with its native look [8].

Pointing out that architecture has always been heavily influenced by past architectural experiences, Newcomb (2007) has pointed to the direct perception of past architectural styles as a diversionary subject, which has been added to the definition of regionalism. This method is used by the ethno-architecture, which has previously looked at some kind of regrettable past. He takes the main difference between regionalism and ethnicity in their view of the future. Ethnicism seeks to revive the characteristics of the past social life in the form of architecture, while regionalism looks at a different and changing future. Ethnicism removes the architect from the scene and believes in the spontaneous creation of architecture, while in regionalism, the architect has an important role in interacting between the design and the location of the generations [9].

\section{Regionalism and Nationalism}

The specific cultural and climatic characteristics, although are taken into account as the two views of regionalization and nationalism, the different motives of these two approaches, in the face of specific regional characteristics, are the basis of the difference between these two attitudes. Berry (2007) defines nationalism as "pride-based regionalism", contrary to tolerance-based regionalism. In nationalism, myths, attributes and abstractions of a place are evaluated separately from the place and become national symbols. Achieving a sense of pride is a feature that is developed by the consolidation of the ruling class to achieve national unity [10].

Harris (2007) defines nationalism as the first type of regionalization by defining two types of regionalism, regionalism, or monopoly against liberalism. In his 
view, limitations of zonality are rooted in distinctions and limitations, and are less indicative of dialectical interaction in clarifying ideas. Such regionalism is an anti-international and anti-progress system. This is despite the fact that freedom regionalism, in addition to emphasizing the existence of characteristics and differences, is positive to the world of culture. According to Harris, the difference between regionalism and nationalism is that the expression of regionalism is based on the expression of freedom. This expression is a picture of the benefits of the new realm, new ways of life, new forms of construction, and new forms of harmony. The human being image uncovers the world in terms of architecture and understands them more than ever that is the picture of liberation, carefreeness and diversity. When architecture depicts diversity, freedom and expansion, can achieve the best regionalism. This architecture recognizes the nationalist expression as a statement of unity, consolidation and anti-diversity, because the word "nation" means united peoples. The goal of nationalist architecture is, more, the unity of the people as their citizens.

Since the nation is essentially a symbol, nationalist architecture must create an image of the qualities that are recognized as the symbol of nationality [11].

Mumford, referring to regionalism developments at the beginning of this century, sees it as a challenge to nationalism and the regionalism of governments. The suppression of regional features in the direction of national unity, so systematically, by modern governments, is the result of this confrontation. According to him, regional groups led to the emergence of a ghost of national disruption, which was fought with them through the educational system and propaganda. He believes that such conflicts are due to the fact that the realities of the region and societies do not coincide with the boundaries and ideological patterns of the national government. Mumford regards regionalism as a cultural and political doctrine as a signifying the inter-regional framework that ultimately leads to global culture. Thus, regionalism, not the emotional and imaginary past, which belongs to the realist future, is a future that will undermine the ineradicable realm of public life, and will devolve upon the ruins of obsessive mythology and the inefficient mechanisms of the mighty state [12].

Regionalism is beyond design on the basis of adaptation or reliance on resources. Regionalization fosters communication with the locality and responds to the needs of native life. Not in conflict with global considerations and preferences, but also to seek out the best of them. The promise of regionalism in architecture means that we understand the realities and diversity of our native places with ease and with a critical look. Regionalism has the capability to put us in continuity with our individual and social history. Ultimately, regionalism provides opportunities for common goals that are perceived here in terms of ecological, economic, and social perception [8].

\section{Theoretical and Typological Typology of Climate Bed Fluidity Approach}

Regionalism is a flexible approach that offers different recommendations in each 
period, according to the circumstances. Flexibility, by adding complementary vocabularies to the term regionalization, makes practical expression and continuity of life possible in the present situation. In this way, the term regionalism finds elegance beyond its verbal meaning and allows for the adoption of various purposes (Shayan \& Kamatiya, 2008, 14). Cole Cohen (2007) explains the above interpretation in the word "fluidity", as one of the features of regionalism. In her opinion, regionalism, like any other school of thought, must be so fluid that it ends up with fruitful social results, despite the inherent contradictions. she states that regionalism must constantly adapt itself to changing conditions and expose itself to each culture. By this definition, Cole Cohen concludes that such an interpretation of regionalism cannot be regarded as a theory, but it may also seem like an approach or perspective [12].

The fluidity and flexibility available in the definitions of regionalism has led various theorists to identify different types of regionalism. However, in all existing definitions, commons such as "paying attention to indigenous and regional characteristics", "responding to genuine needs rooted in culture and traditions", "attention to the past based on choice and not coercion", and "attention to today's needs and conditions" exist, but there are distinct differences between them in terms of practical arrangements and mechanisms for confronting the place and the region. These differences are due to the different influences that regionalism accepts from its theories and views. Fluency of dominant architectural ideas such as classicalism, modernism, international style, postmodernism, and theories governing human relationships such as globalization, and multi regionalism in recent years have raised regionalism from different aspects in architecture literature [13].

The description of regionalism and recognition of the theoretical grounds and their practical procedures can redefine the contradictions and ambiguities in explaining the regionalization approach. What matters at any time is how has been the link between architecture and cultural features. Categorizing existing approaches can make future predictions relevant to changing variables governing global thought. The answer to the question of how today regionalism can respond to the concerns and identity of peoples facing a world without borders is the time-based analysis of existing attitudes. Several phrases derived from such interactions indicate the potential dynamics of regionalism. The basis of this dynamics is hidden in the dynamic interpretations of the word region [14].

\section{Hospital}

The hospital is a social institutional gradually emerged in the history of social life in line with the necessity of survival of humans, and the return to health, along with the evolution and development of science and technology, and changes in the way of life as a requirement for human beings. Therefore, the history of the hospital is interwoven with medical history, and in fact the growth and development of the hospital is influenced by the progressive development of 
medical science and technology, especially in the current century [15].

Medicine is among the most important social-cultural institutions that human beings directly interact with, due to its association with human life. Hence, there is a long history as part of human needs and his desire for survival. Historical evidence suggests that medical developments in Babylonia began 6000 years ago, 4000 years before Greece [16]. The Babylonian religion was based on the existence of numerous gods. Thus, hospitals gradually developed to fight diseases, especially those that were not treated either at home or through a referral to a surgeon. Otherwise, the need for the use of new and complex technology enabled easier and more affordable use of them in hospitals, as thus health centers are becoming more and more widespread [15].

\section{A Glance at Hospitals Activities}

Continuity of life is a function of a society and a system of any kind, material or non-material to carry on its work from the community, and the process of movement of the organization is influenced by the interaction with other social institutions in society, and the society and its institutions have their own historical background, culture and values differentiate their values from other communities. Thus, a hospital should have sufficient facilities, be able to expand, and in addition to social conditions, it should be well accountable to the climatic and native conditions and in the face of changes over time [15].

The first years of the $21^{\text {st }}$ century have been associated with tremendous human life events. These developments have influenced globalization, economics, culture, social relations and human life, and have reflected these relations in space and, in particular, in cities. Undoubtedly, the most significant characteristic of this century is the opening of new perspectives in human settlements and the unprecedented concentration of populations in metropolises. Generally, the deployment of many urban elements, and, in general profit, is more dependent on economic mechanisms and liberalization, but public nonprofit elements of cities can be entirely left to the economic mechanisms of the market, and to compensate for the market inefficiencies, it has to resort to public interests, and health services are among them. The main task of urban planners and decision makers is to determine the optimal location accessible for all urban residents. In addition, urban planners are trying to optimize the distribution of service centers in urban environments, and this distribution is proportional to the distribution of the population and with demand in different parts of the city [17].

Throughout history, hospitals and health centers have developed based on the ability to treat diseases and protect the health of patients, which means treating illness and, in cases where therapies are not available, to relieve pain in patients. This attention has been challenged in recent years because of the reversal of increased levels of chronic diseases that is seen with age in the population needs to be effective [18].

In order to fully understand the health benefits of patients, employees and the 
community not only need to improve health in the form of limited projects, but should be integrated into a comprehensive and integrated approach to the management systems (quality) of hospitals and health centers. This process involves steps 1-Commitment, 2-Resources, 3-Communication, 4-Planning Activities, 5-Evaluation, 6-Training, 7-Research, 8-Sustainability: Applying methods to measure and maintain health outcomes, achievements and health benefits for patients, staff and community over time. These changes including clinical outcomes in terms of mortality, morbidity and quality of life, the health, health status of employees, the satisfaction of service users or employees, health literacy and population health are measurable. 9-Networking: At all levels (local, regional, national and international) to share in the best activities and strategies to improve the quality and wellbeing of health care centers [18].

\section{Compatibility City Layer (Appropriate Adjacent Applications)}

Compatibility means matching, coordinating and absence of disturbance between two types of urban utilities, which are among the stages of urban spatial analysis. According to urban planning, each utilization in the city is confronted with some uses, and coordinated with others and this compatibility and incompatibility are defined with factors such as noise pollution, air pollution, existing and proposed accesses, and the like [17].

\section{Hospital Architecture}

The shape of a hospital is heavily influenced by how it is accessed and its passageways; therefore, it is decided in this case that a shape like the spine with branches to be taken with a central core with access to other parts [19].

While hospitals were deliberately designed for medical and surgical purposes, today, one can see a change in the way towards humanism in hospital settings. Today's hospitals are more like a hotel. The presence of living space is more important than the plans of cold hygiene in past hospitals. The duration of hospitalization and the patient's stay is steadily shortened, and the interest in a one-bed or two-bed rooms (for privacy of patients) has increased [19].

\section{Agile Hospital}

Hospitals today have entered a competitive environment and should be responsive to their clients. Hospitals as complex organizations should be learners for success in order to be able to respond faster, cheaper, and more efficiently to patients' requests. The hospital's agility reflects hospital accountability when faced with internal and external changes, and agile hospitals have the ability to compete with other hospitals to provide patients with services. By increasing competition and unpredictable changes in business, organizations are taking competitive advantage in achieving organizational goals and success. The work required to act as an agile organization. An agile organization is fast, consistent and ro- 
bust in the face of sudden changes, and responses well to new market opportunities and customer requirements flexibly [20].

Human factor is the factor that plays a decisive role in the provision of health services, and the technology plays a lesser role in providing services. Agility is in fact a necessity to survive against competitors, under changing environments to deal with the challenges of fast delivery of products and services, customer quality and customer service. Service organizations have faced dramatic changes since the beginning of the $21^{\text {st }}$ century. The severity of these changes has been to the extent that service organizations have encountered new challenges, and ignoring these challenges has greatly impacted the survival and success of organizations. Some of these changes are Markets Globalization, the customers diversely need for services, the customers' demand for meeting their demand individually, the emphasis of the organizations on the introduction of new services and the focus on their marketing. These factors and some other factors have led the service organizations to operate today in an environment with change as its main feature. The rapid pace of change in the world's hospitals, the public hospitals has no choice but to coordinated among all factors involved in the production of services, suppliers and distributors in the shortest possible time to the patient to succeed in a rich market. Agility is one of the supply chain paradigms, which can provide a suitable platform for expediting the process of operations and business processes and identifying its changes at each stage of the supply chain, thus reducing additional and repeated operations that slow down the supply chain process. The paradigm can improve the chain quality and flexibility by increasing speed and accuracy, reducing customer response times, reducing rework, and so forth [20].

\section{Conclusion}

The introduction of climatism in the context of building hospitals is to be a key factor in influencing the design of these centers and can improve the process of providing optimal services to clients by these units. A range of factors from the community beliefs to the natural factors all govern the context of the construction of the hospital. The impact of natural climatic factors in the hospital's body assessment shows high impact of special conditions in public opinion. It is suggested that the construction of this body should be in such a way that it best corresponds to the texture of the site and to attract clients. If it is recommended that the fluidity of the movement be created within the building, it would be preferable to promote the sense of belonging to the building for users. Various factors are involved in the process of construction of healthcare centers such as social factors, community structure, the type and speed of technology development in the region, the climate of the region, the extent of the need for therapeutic activities in the region and many other factors. One of the topics discussed in relation to the construction of hospitals is the discussion of the climate and the overall context of the region with regard to the residents. The view of residents 
on the monuments built on the historical background of the community can be referred to regionalism, nativism, a traditional (Traditional architecture), in other words, traditionalism, ethnocentrism, nationalism, and climatism. The link between the community and a wide range of users with such buildings is important because of the fluidity of the movement on public utilities. Notably, with the advancement of technology and the emergence of new technologies, the process of building construction is subject to change in line with the contemporary processes. However, if these changes, whether small or large, are applied through the establishment of a link between the community and society, it will integrate the community and the region. Attention to the climate and the type of attitudes present in the community, and balancing these attitudes and human resources create a fluid environment in which everything is done well. Although making adaptations in such centers is difficult in terms of their complexity of use, the alignment of these units with climatic conditions can to some extent reduce the burden on public opinion.

\section{Recommendations and Suggestions}

Given the importance of the climate in the process of hospital construction and its impact, the following points are suggested:

- Maintaining the traditional view of the community towards the building so that it improves the performance of these centers; since the discussion of treatment has a kind of anxiety in the minds of the patients, if the traditional look is included in the building, in terms of the establishment of the initial bond with the traditional view can reduce this distress to some extent, because tradition in collective thoughts is something that is holy and linked to divine inspiration. It is suggested that by creating this link, there is a ground that makes its users in an environment not separated from their habitat.

- Maintaining the relationship between humans and the environment; it is recommended that the building be in such a way as to be in an environment that is inseparable from it; as the earth and all of its natural elements remain entirely or partly integrated in to the same harmony. It is recommended that what is added to this collection is also created in harmony with the whole complex. Thus, the patient arrived at a treatment center find himself in a familiar place. Therefore, a native attitude and some modifications of the national attitude can help.

- Using native materials; aligning large public buildings such as hospitals with residential buildings in the local and regional dimension is not a simple thing. The design and construction of residential buildings have a deep relationship with the thoughts and culture of the community, with added traditional materials and facilities, while public buildings are generally advised to move towards the efficiency of day-to-day technologies, therefore, one of the possible constraints on the construction health centers would be the use of indigenous materials, but it is recommended to use them in terms of linking 
these centers with other buildings.

- The need to use new technologies in health care centers; adapting and integrating the use of indigenous materials and productivity of new technologies is recommended, in which the two factors complement each other.

- Locating in accordance with the texture of the region; the decision at the location of the healthcare center is made with regard to various factors such as rapid access (i.e., the agility of a healthcare center), the shape of the land, the type of soil in the area due to the breadth of the building, the impact of natural factors such as wind and light, construction materials in the region, etc., points out the necessity of emphasizing the attitude of climatism.

\section{Conflicts of Interest}

The authors declare no conflicts of interest regarding the publication of this paper.

\section{References}

[1] Rudolph, A.M. (1985) Distribution and Regulation of Blood Flow in the Fetal and Neonatal Lamb. Circulation Research, 57, 811-821. https://doi.org/10.1161/01.RES.57.6.811

[2] Shultz, P.A., Kamal, R.N., Daniels, A.H., Di Giovanni, C.W. and Akelman, E. (2015) International Health Electives in Orthopaedic Surgery Residency Training. The Journal of Bone and Joint Surgery. American Volume, 97, e15.

[3] Onlen, Y., Duran, N., et al. (2007) Antibacterial Activity of Propolis against MRSA and Synergism with Topical Mupirocin. The Journal of Alternative and Complementary Medicine, 13, 713-718. https://doi.org/10.1089/acm.2007.7021

[4] Imani, A., Mansoori, F. and Mooei, M. (2016) Social and Cultural Impact Assessment of Geometric Correction of Urban Passages (By Studying the Geometric Correction of Passages in District 18 of Tehran). Quarterly Journal of Research and Urban Planning, 7, 85-104.

[5] Palmer, J., Cooper, I. and van der Vorst, R. (1997) Mapping out Fuzzy Buzzwords-Who Sits Where on Sustainability and Sustainable Development. Sustainable Development, 5, 87-93. https://doi.org/10.1002/(SICI)1099-1719(199708)5:2<87::AID-sd70>3.0.CO;2-Z

[6] Capon, F., Novelli, G., et al. (1999) Searching for Psoriasis Susceptibility Genes in Italy: Genome Scan and Evidence for a New Locus on Chromosome. Journal of Investigative Dermatology, 112, 32-35. https://doi.org/10.1046/j.1523-1747.1999.00471.x

[7] Mumford, D. and Musili, C. (2007) Tata Lectures on Theta II. Volume 43, Springer, New York. https://doi.org/10.1007/978-0-8176-4578-6

[8] Cannizzaro, G., Leone, M. and Esposito, M. (2007) Immediate Functional Loading of Implants Placed with Flapless Surgery in the Edentulous Maxilla: 1-Year Follow-up of a Single Cohort Study. International Journal of Oral \& Maxillofacial Implants, 22, 87-95.

[9] Newcomb, M. (2012) Sustainability as a Design Principle for Composition: Situational Creativity as a Habit of Mind. College Composition and Communication, 63, 593-615. 
[10] Berry, J.K., Detgado, J., Khosla, R. and Pierce, F.J. (2003) Precision Conservation for Environmental Sustainability. Journal of Soil and Water Conservation, 58, 332-339.

[11] Harris, P.R., Mayle, K., Mabbott, L. and Napper, L. (2007) Self-Affirmation Reduces Smokers' Defensiveness to Graphic on-Pack Cigarette Warning Labels. Health Psychology, 26, 437-446. https://doi.org/10.1037/0278-6133.26.4.437

[12] Colquhoun, I. (2007) Design out Crim. Routledge, London. https://doi.org/10.4324/9780080470092

[13] Daad, Y., Emami, H., et al. (2010) Values and Environmental Functions of Green Roofs in Sustainable Urban Development. The First Sustainable Urban Development Conference, Guilan University, Guilan.

[14] Kamel Nia, H., Fereydouni, F., et al. (2016) The Impact of Sustainable Architecture on Reducing the Syndrome of a Patient's Building. International Conference on Modern Horizons of Architecture and Urbanism, Tehran.

[15] Ahmadi, Y. (2007) Rehabilitation Hospital. Master's Thesis, Azad University of Hamedan, Hamedan.

[16] Moshabaki Esfahani, A. (2010) Architectural Design Guide for Healthcare Buildings (Hospitals Architecture). Parham Publication, Tehran.

[17] Ebrahim Zadeh, E., Ahamad Nejad, M., et al. (2010) Local-Spatial Planning and Organizing of Health Services Using GIS. Human Geography Research, 73, 39-58.

[18] Nik Pazhooh, A. and Samadi, B. (2011) International Network of Health Promoting Hospitals and Health Centers: Integration of Health Promotion in Hospitals and Health Centers. Publications of the Institute for Preventive and Health Promotion of Contemporary Iranians.

[19] Ernst, P.N. (2013) Architectural Information (Translated by Mozafari Torshizi, H. and Pahizkar, T.). Azadeh, Tehran.

[20] Abdi Talar Poshti, M., Mahmoudi, G.H. and Jahani, M.A. (2016) Factors Affecting the Agility Supply Chain of Iranian Hospitals. Journal of Health Administration, 19, 7-18. 